\title{
A Comprehensive Database and Subjective Evaluation Methodology for Quality of Experience in Stereoscopic Video
}

\author{
Lutz Goldmann $^{a}$, Francesca De Simone ${ }^{a}$ and Touradj Ebrahimi ${ }^{a}$ \\ ${ }^{a}$ Ecole Polytechnique Federale de Lausanne (EPFL), CH-1015 Lausanne, Switzerland
}

\begin{abstract}
While objective and subjective quality assessment of 2D images and video have been an active research topic in the recent years, emerging 3D technologies require new quality metrics and methodologies taking into account the fundamental differences in the human visual perception and typical distortions of stereoscopic content. Therefore, this paper presents a comprehensive stereoscopic video database that contains a large variety of scenes captured using a stereoscopic camera setup consisting of two HD camcorders with different capture parameters. In addition to the video, the database also provides subjective quality scores obtained using a tailored single stimulus continuous quality scale (SSCQS) method. The resulting mean opinion scores can be used to evaluate the performance of visual quality metrics as well as for the comparison and for the design of new metrics.
\end{abstract}

Keywords: 3DTV, database, quality of experience, subjective quality evaluation

\section{INTRODUCTION}

The introduction of three dimensional television (3DTV) on the public consumer market is believed to be just a matter of time and has been compared to the transition from black-and-white to color TV. To be a success, both visual quality and comfort must at least be comparable to conventional standards to guarantee a strain free viewing experience. Since 3DTV involves both 2D and 3D visual perception new distortions have to be considered beside the classical ones.

The quality of 3D content may be affected by artifacts arising from each of the stages within a typical 3D processing chain: ${ }^{1}$

Creation: For the creation of 3D image and video content three different approaches can be distinguished. First, such content can be captured by two or more synchronized cameras in a multiview setup and an augmented by depth information captured with a special sensor. Second, 3D content can be rendered from a 3D model of the scene using techniques from computer graphics. Finally, existing 2D content can be converted into 3D content using manual or automatic video processing techniques. The positioning of the cameras or the selection of the rendering parameters has a direct influence on the perceived depth of a scene. Unnatural correspondences between two stereo images or videos lead to a number of very disturbing artifacts.

Representation: Different scene representations have been proposed for 3D image and video including the multiview representation where each view is represented as an individual image, and the image+depth representation where a scene is represented by an image and a depth map from which the individual views can be rendered. Each of these representations may cause specific artifacts such as vertical parallax for multiview and disocclusions for image+depth.

Coding: Typical image and video compression schemes for 2D and 3D exploit the spatial, temporal redundancies (lossless) and irrelevancies (lossy) to compress the source signal. This may lead to 2D artifacts such as blocking, blurring, and ringing, which may also influence the 3D perception.

Further author information: (Send correspondence to Lutz Goldmann)

Lutz Goldmann: E-mail: lutz.goldmann@epfl.ch, Telephone: +41 216934807

Francesca De Simone: E-mail: francesca.desimone@epfl.ch, Telephone: +41216934621

Touradj Ebrahimi: Email: touradj.ebrahimi@epfl.ch, Telephone: +41 216932606 


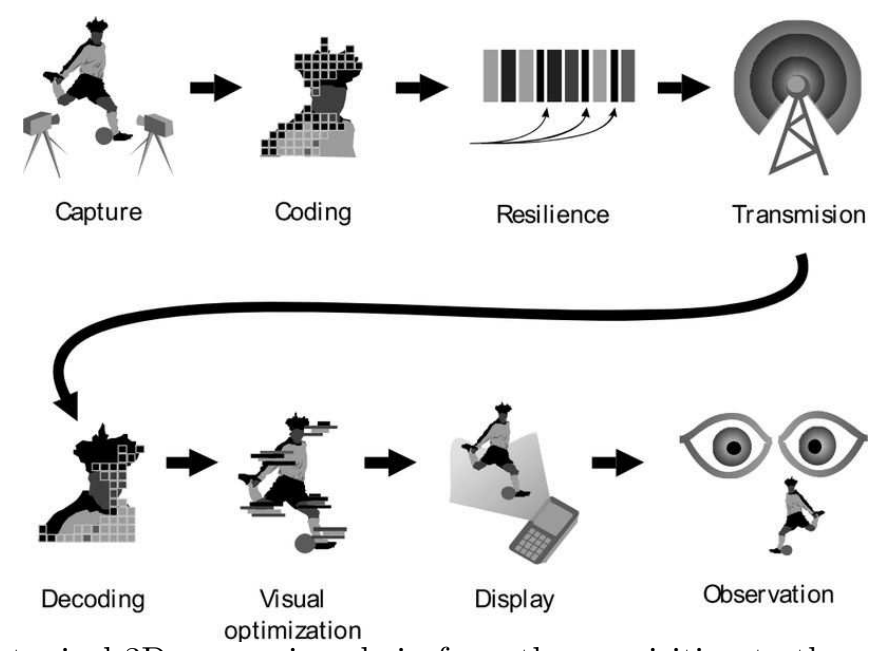

Figure 1: Overview of a typical 3D processing chain from the acquisition to the restitution of a 3D scene. ${ }^{1}$

Transmission: One of the most common problems in digital transmission is packet loss, which may influence the image and video quality considerably. Most of the error resilience and concealment techniques have been designed for 2D video, but they may influence the 3D quality as well.

Restitution: Various technologies have been proposed to deliver stereoscopic images and videos through partitioned optical channels to the viewer's eyes, including anaglyph, polarization, shutter and auto-stereoscopic. Each of these approaches has its specific artifacts which are also highly scene dependent.

While the creation and restitution stages have a direct influence on the 3D perception, the other stages may also introduce artifacts leading to a reduction in the perceived 3D quality.

Several parameters have an influence on the 3D effect of the captured stereoscopic video including the interocular distance between the camera lenses, the angle between the cameras which can be either parallel or converged and the distance of the real scene. In order to provide the observer with an optimal 3D effect it is important to understand the influence of these parameters on the quality of the perceived 3D content.

While the effects of the different acquisition parameters on the 3D perception have been studied and described before, their influence on the perceived quality for a human observer has not been assessed quantitatively. Furthermore, there is a lack of comprehensive stereoscopic image and video datasets that support the development and evaluation of 3D processing techniques. The objective of this work is to develop a comprehensive 3D image database with associated subjective quality scores comparable to the LIVE Image Quality Database* and the Tampere Image Database (TID) $2008^{\dagger}$ for $2 \mathrm{D}$ images. Therefore, we have created a stereoscopic video dataset with various contents captured with different acquisition parameters. Given that dataset we have conducted extensive subjective tests to study the influence of the acquisition parameters on the perceived 3D quality.

The paper is organized as follows. Section 2 discusses the novel stereoscopic video database including the initial requirements, the acquisition and the postprocessing of the videos, and provides a description of the individual scene characteristics. Section 3 describes the goal and procedure of the subjective quality evaluation. Section 4 discusses the statistical analysis of the subjective quality scores and discusses the results of the subjective quality test. Finally, section 5 summarizes the major findings and proposes directions for the use and extension of the proposed database.

${ }^{*}$ http://live.ece.utexas.edu/research/quality/subjective.htm

${ }^{\dagger}$ http://www.ponomarenko.info/tid2008.htm 


\section{DATABASE}

\subsection{Requirements}

In order to make a 3D video database suitable for the development and evaluation of a complete 3D processing chain and a wide variety of applications, it has to fullfil several requirements. The following list of requirements is inspired by the recent calls of MPEG for $\mathrm{FTV}^{2}$ and $3 \mathrm{DTV}^{3}$ test material:

- The cameras shall be in a $1 \mathrm{D}$ parallel arrangement.

- The type of cameras should be reported with data sheets and intrinsic information if possible.

- The temporal synchronization of the multiple cameras should be as accurate as possible.

- The minimum spatial resolution should be VGA (640x480) and the minimum spatial resolution 24 fps. However, higher spatial resolutions and temporal resolutions are desirable to simulate current and future media standards.

- Color consistency between the multiple views is very important and the cameras should be white balanced.

- The content should be representative and challenging with variations in texture, moving objects, camera movements, scene (depth) structures and lighting conditions.

As it will be shown below, most of the requirements are addressed by the proposed database. Since only two views and no depth information is provided, the database may not be suitable for free-viewpoint or 3D video applications. However, stereoscopic videos can be seen as the native representation of a single view of a 3D scene and is widely used for 3D processing.

\subsection{Acquisition}

For acquiring high quality stereoscopic images and video the following aspects have to be considered: ${ }^{4}$

Matching cameras: Two identical cameras with the same firmware and settings should be used for complete interchangeability.

Matching optics: Both camera have to be optically matched in focal length and focus point. Since zoom lenses may differ only the extreme ends of the zoom range should be used.

Matching geometry: Both cameras have to be in perfectly controlled relative position to each other. From all possible translations and rotations, only translation along the horizontal axis and rotation along the vertical axis are allowed.

Matching photography: Full manual mode of the cameras should be used to ensure matching in white balance, sensitivity, shutter speed, aperture, gain, and framerate.

Synchronization: Accurate synchronization between the two cameras is essential and can be achieved by using a single remote control or electronically synchronized cameras.

Considering the different aspects mentioned above, we have built the stereo camera setup shown in figure 2, which consists of two identical HD camcorders (Canon HG-20) and an adjustable stereo mount. The mount ensures that optical axes of the cameras are parallel and supports the continuous adjustment of the camera distance in the range $7-50 \mathrm{~cm}$. To ensure matching of the focal length the wide angle end of the zoom lens with a focal length of $43 \mathrm{~mm}$ has been used. In order to match the cameras with each other the focal length, white balance and shutter speed have been set manually. The synchronized operation of the two camcorders is ensured through the use of a single remote control. The camcorders support the capture of progressive video with a resolution of 1920x1080 pixels and a frame rate of $25 \mathrm{fps}$. The videos are stored in AVCHD format which uses MPEG-4 AVC/H.264 for video compression and Dolby AC-3 for audio compression. The videos are captured using the MXP mode with a constant bitrate of 24 Mbps which delivers the highest visual quality. 


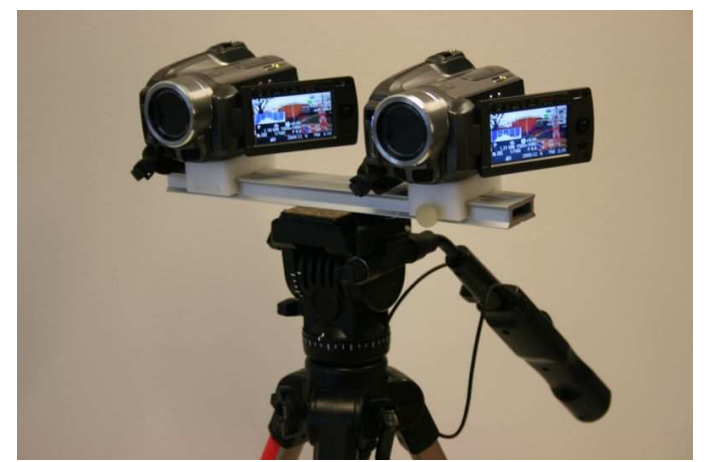

Figure 2: HD stereo camera setup consisting of two identical camcorders and an adjustable stereo mount.

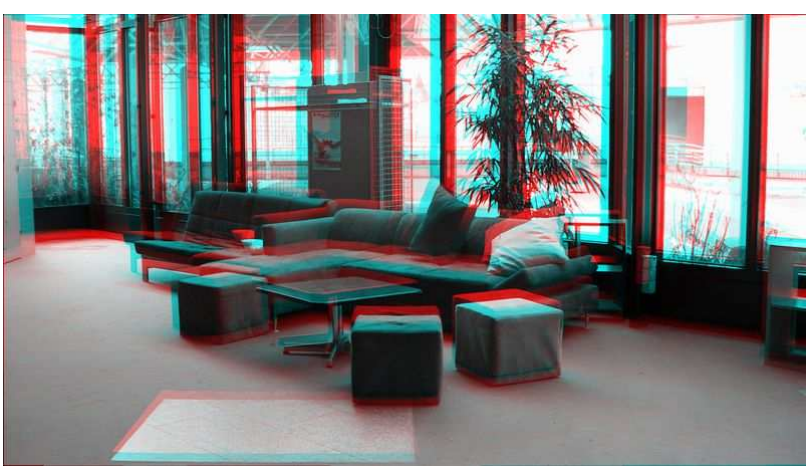

(a) Before

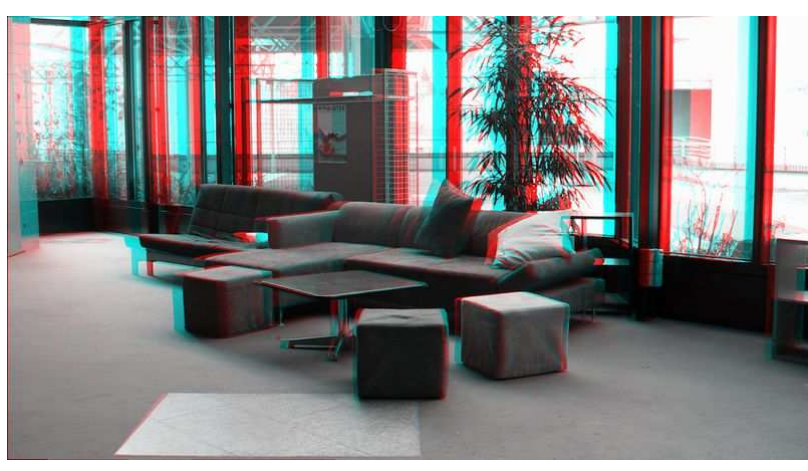

(b) After

Figure 3: Result of the geometrical alignment for a stereo image pair (anaglyph view).

\subsection{Postprocessing}

Because no 3D camera setup is perfect, a postprocessing step is needed to correct small calibration errors. This includes geometrical and temporal alignment and color adjustment. ${ }^{4}$

\subsubsection{Spatial alignment}

In a stereoscopic camera setup, spatial distortions may be caused within the individual cameras (e.g. barrel/ pincushion distortion) or by the camera setup and calibration (e.g. relative positions). The spatial alignment focuses on the correction of the inter-camera distortions which are specific for the parallel camera setup.

The goal of the spatial alignment is to compensate small vertical disparities caused by the camera setup and adjust the depth position to avoid stereo window violations. This is achieved by applying a relative vertical and horizontal translation between the video pairs based on point correspondences. For a reliable adjustment of the depth position the control points for the nearest object are manually selected.

\subsubsection{Temporal alignment}

In a stereoscopic camera setup temporal mismatch may occur if the cameras are not shutter-synchronized. Therefore the left and the right frames are not captured simultaneously but slightly shifted in time. While dense depth estimation requires precise synchronization, the human visual system can cope with small amounts of time shift without a decrease of perceptual quality.

In order to avoid temporal mismatch a remote control has been used to control both camcorders simultaneously. The synchronization accuracy has been checked manually and was typically below $40 \mathrm{~ms}$ which is equal to 2 frames. To eliminate the influence of temporal mismatch on the subjective quality the videos were manually aligned according to the temporal offset. 


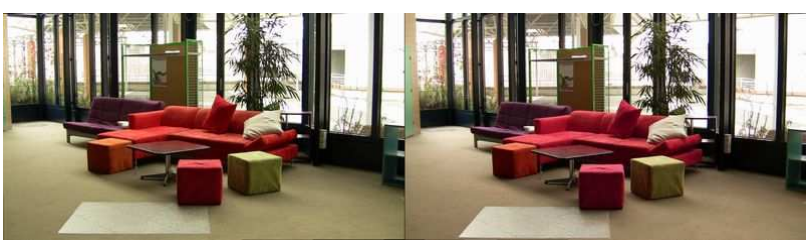

(a) Before

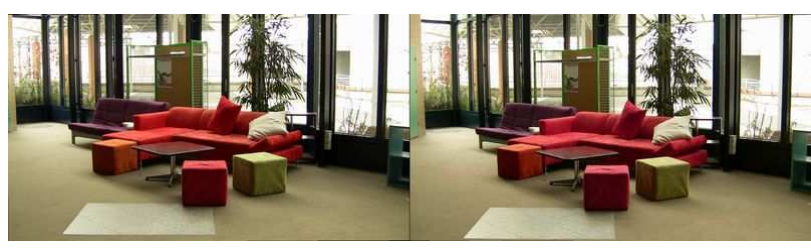

(b) After

Figure 4: Result of the color adjustment for a stereo image pair (left-right view).
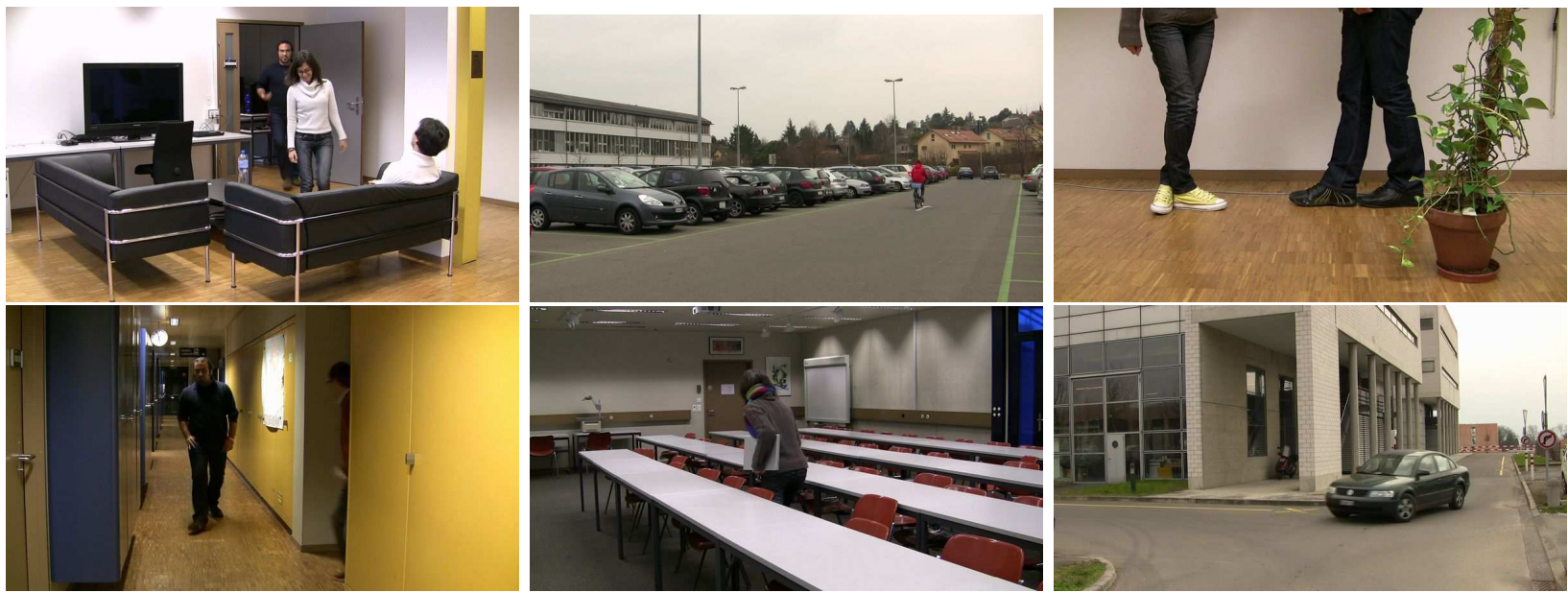

Figure 5: Visual samples of the different scenes considered within the database.

\subsubsection{Color adjustment}

Even with a manual control of white balance and exposure, luminance and chrominance components may vary globally between the different views. These discrepancies may originate from the use of heterogeneous cameras, calibration errors and appearance changes due to the different viewing angles. The goal of color adjustment step is to correct these color differences between the two stereo images.

Histogram matching as proposed by Hekstra et al. ${ }^{5}$ is used to adapt the right camera view to the left camera view. This method assumes that a good fit of the distorted image to the reference image can be obtained by adapting the cumulative histogram. It makes no assumptions about the type of distortion such as brightness or contrast variations and considers nonlinear mappings. Since the correction is applied to the entire image, it is especially useful to correct global luminance and chrominance differences.

\subsection{Description}

The proposed database contains stereoscopic videos with a resolution of 1920x1080 pixels and a frame rate of $25 \mathrm{fps}$. Various indoor and outdoor scenes with a large variety of colors, textures, moving objects and depth structures have been captured. Each of the scenes has been captured with a static camera and different camera distances in the range $10-50 \mathrm{~cm}$. Since the acquisition was done in a sequential way the content of a single scene may vary slightly across the different camera distance. However, the general 2D (color, texture, motion) and 3D (depth) characteristics are preserved.

A subset of 6 scenes, shown in figure 5, with different characteristics has been selected for the subjective quality evaluation within this paper.

Table 1 provides an overview of the selected scenes together with the 3D characteristics such as near distance $n$ and far distance $l$, and the maximum permissible camera distance $b$. The latter can be theoretically computed based on a simplified Bercovitz equation ${ }^{6}$ as

$$
b=\frac{p}{f} \cdot \frac{l \cdot n}{l-n}
$$


Table 1: Overview of the different scenes and their characteristics.

\begin{tabular}{rlrrr}
\hline Id & Title & Near $(\mathrm{m})$ & Far $(\mathrm{m})$ & Distance $(\mathrm{cm})$ \\
\hline 1 & sofa & 3 & 6 & 17 \\
2 & bike & 10 & 150 & 30 \\
6 & feet & 2 & 4 & 11 \\
8 & hallway & 2 & 20 & 6 \\
11 & notebook & 3 & 10 & 12 \\
12 & car & 8 & 120 & 24 \\
\hline
\end{tabular}
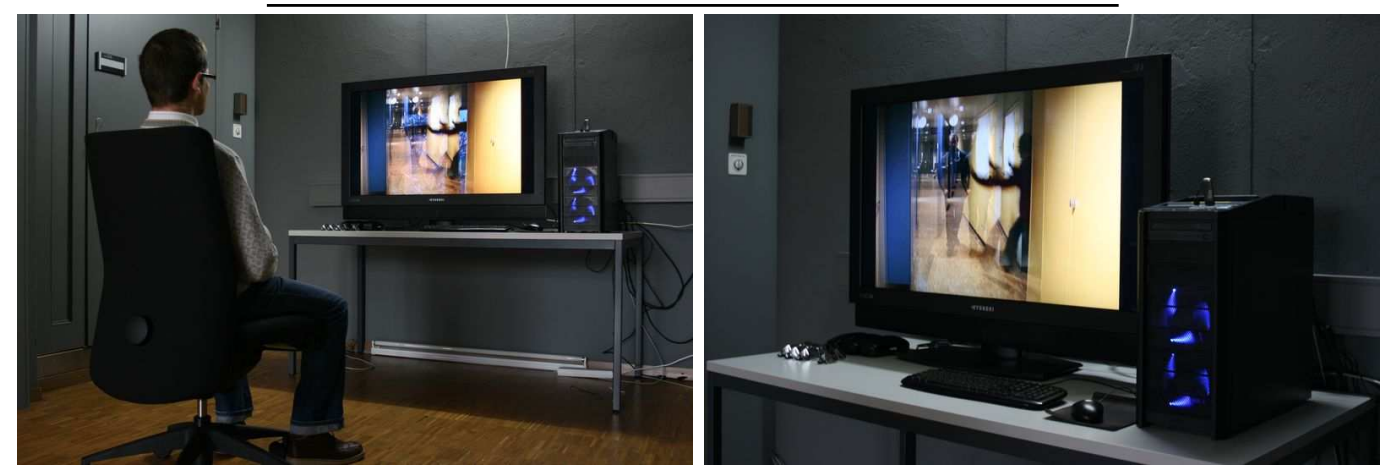

Figure 6: Quality test laboratory and 46" polarized 3D display used for the subjective quality evaluation.

with the focal length $f=43 \mathrm{~mm}$ and a typical parallax limit $p=1.2 \mathrm{~mm}$. The simplification of the Bercovitz equation is valid for $f \ll n$, which is true for all the scenes in the database.

\section{METHODOLOGY}

\subsection{Equipment}

The subjective test was conducted at the Multimedia Signal Processing Group (MMSPG) quality test laboratory at EPFL (shown in figure 6), which is compliant with the recommendations for subjective evaluation of visual data issued by ITU-R. ${ }^{7}$ The laboratory is setup in a way to assure the reproducibility of results by avoiding involuntary influence of external factors.

A 46" polarized stereoscopic display (Hyundai S465D) with a native resolution of 1920x1080 pixels has been used to display the test stimuli. The monitor was calibrated using an EyeOne Display2 color calibration device according to the following profile: sRGB Gamut, D65 white point, $120 \mathrm{~cd} / \mathrm{m}^{2}$ brightness and minimum black level. The room is further equipped with a controlled lighting system that consists of neon lamps with $6500 \mathrm{~K}$ color temperature. The illumination level measured on the screen is 30 lux and the ambient black level is $0.5 \mathrm{~cd} / \mathrm{m}^{2}$.

The experiments involved only one subject per session assessing the test material. The subject was seated in line with the center of the monitor, at a distance of approximatively $2 \mathrm{~m}$ which is equal to the height of the screen multiplied by factor 3 as suggested in the ITU-R BT.710 recommendation ${ }^{8}$ for HDTV.

\subsection{Observers}

Twenty subjects (6 female, 14 male) participated in the test. All of them are non-expert viewers with a marginal experience of 3D image and video viewing. The age distribution ranged from 24 to 37 with an average of 27 .

All the subjects were screened for visual acuity using the Snellen chart and color vision using the Ishihara test. Furthermore, the stereo vision was tested according to the ITU recommendation. ${ }^{9}$

\subsection{Stimuli}

For the subjective evaluation the stereoscopic video databased described in section 2 has been split into a training set with 1 scene (stairs) and a testing set with 6 scenes (sofa, bike, feet, hallway, notebook, car). For each of the scenes 5 different stimuli have been considered corresponding to different camera distances $(10,20,30,40$, $50 \mathrm{~cm}$ ). 


\subsection{Procedure}

The subjective evaluation of $2 \mathrm{D}$ visual quality according to standardized methods has a long history. Several recommendations have been issued by the International Telecommunication Union (ITU) including the widely used ITU-R BT.500. ${ }^{7}$ It describes methods for the subjective quality assessment of standard definition television (SDTV) pictures. The most prominent methods are the double stimulus continuous quality scale (DSCQS), the double stimulus impairment scale (DSIS) and the single stimulus continuous quality evaluation (SSCQE). While DSCQS and DSIS can be used for a direct comparison of impaired and unimpaired stimuli, SSCQE is suitable to assess the quality without a reference. With respect to the subjective quality evaluation of future multimedia data such as high definition (HDTV) and 3-dimensional TV (3DTV) the same methods are recommended in ITU-R BT. $710^{8}$ and ITU-R BT. $1438,{ }^{9}$ respectively.

Since the optimal acquisition settings for 3D content may vary depending on the scene, the display and the observer, it is difficult to select one of the stimuli as a reference. Therefore, a single stimulus (SS) method has been adopted for the subjective quality evaluation. In order to determine the influence of the camera distance on the 3D quality, a continuous quality scale with 5 levels (excellent, good, fair, poor, bad), as described in ITU-R BT. $500,{ }^{7}$ has been used.

During the training session the subjective test methodology was introduced to the subjects and the range of quality levels was explained through a set of training stimuli. The stimuli have been selected by an expert viewer in such a way that each quality level is represented by an example and that the full range of quality levels within the set of test stimuli is covered. The training stimuli were presented in the same way as the test material to familiarize the subjects with the methodology.

During the testing session the subjects evaluated the quality of the 30 test stimuli, which are displayed in random order. Each stimulus is shown once with a duration of $10 \mathrm{~s}$ and a $5 \mathrm{~s}$ break between the stimuli, during which the subjects provide their scores.

\section{ANALYSIS AND RESULTS}

\subsection{Outlier detection}

The screening of subjects was performed according to the guidelines described in section 2.3 .1 of annex 2 of ITU-R BT. 500-11 recommendation. ${ }^{7}$

First, for each stimulus, it is tested whether the distribution of scores across subjects is normal or not. This is done by calculating the kurtosis coefficient of the distribution: if the coefficient is between 2 and 4 , the distribution is assumed to be normal. Then, the score of each observer is compared with an upper and a lower threshold computed as the mean value plus and minus the standard deviation associated to that stimulus (times two, if normal, or times 20, if non-normal). For each subject, every time his/her score is found above the upper threshold a counter, $P_{i}$, is incremented. Similarly, every time his/her score is found below the lower threshold, a counter, $Q_{i}$, is incremented. Finally, the following two ratios are calculated: $P_{i}+Q_{i}$ divided by the total number of scores from each subject for the whole session, and $P_{i}-Q_{i}$ divided by $P_{i}+Q_{i}$ as an absolute value. If the first ratio is greater than $5 \%$ and the second ratio is less than $30 \%$, then subject $i$ is an outlier and all his/her scores are discarded.

Using the outlier detection described above, 3 of the 20 subjects have been discarded as outlier. Thus the statistical analysis is based on the scores from 17 subjects.

\subsection{Score computation}

After the outlier removal, the mean opinion score is computed for each test condition $j$ as:

$$
M O S_{j}=\frac{\sum_{i=1}^{N} s_{i j}}{N}
$$

where $N$ is the number of valid subjects and $s_{i j}$ is the score by subject $i$ for the test condition $j$.

The relationship between the estimated mean values based on a sample of the population (i.e. the subjects who took part in our experiments) and the true mean values of the entire population is given by the confidence 

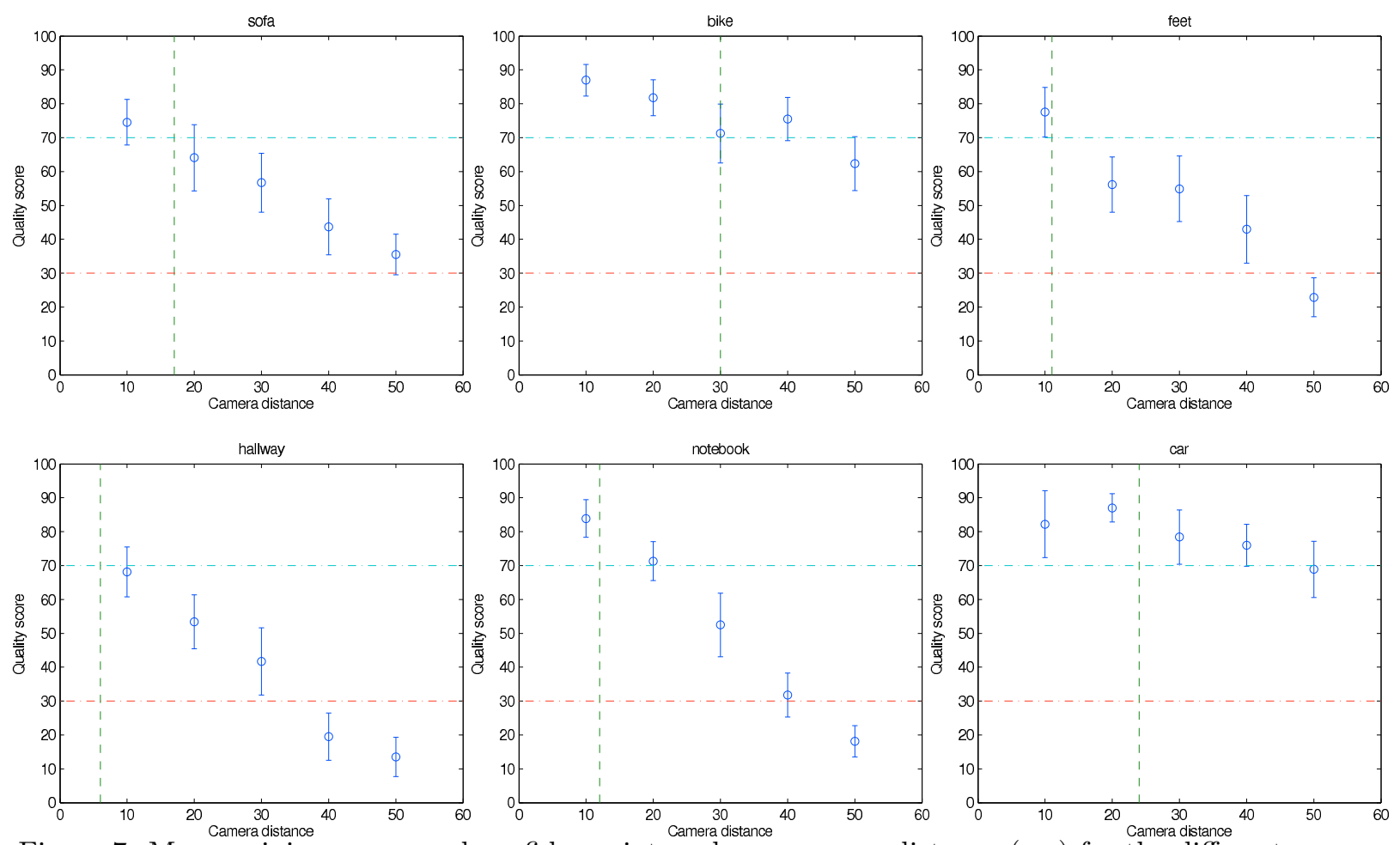

Figure 7: Mean opinion scores and confidence intervals vs. camera distance $(\mathrm{cm})$ for the different scenes.

interval of the estimated mean. Due to the small number of subjects, the $100 \times(1-\alpha) \%$ confidence intervals (CI) for mean opinion scores are computed using the Student's t-distribution, as follows:

$$
C I_{j}=t(1-\alpha / 2, N) \cdot \frac{\sigma_{j}}{\sqrt{N}}
$$

where $t(1-\alpha / 2, N)$ is the t-value corresponding to a two-tailed t-Student distribution with $N-1$ degrees of freedom and a desired significance level $\alpha$ (equal to 1-degree of confidence). $N$ corresponds to the number of subjects after outlier detection, and $\sigma_{j}$ is the standard deviation of a single test condition across the subjects. The interpretation of a confidence interval is that if the same test is repeated for a large number of times, using each time a random sample of the population, and a confidence interval is constructed every time, then $100 \times(1-\alpha) \%$ of these intervals will contain the true value. We computed our confidence intervals for an $\alpha$ equal to 0.05 , which corresponds to a significance level of $95 \%$.

Figure 7 plots the mean opinion scores and confidence intervals vs. the camera distance for each of the individual scenes. The maximum theoretical camera distances from table 1 are also shown as a dashed vertical line. In general the small size of the confidence intervals shows that the complexity of the subjective evaluation tasks was appropriate and that the ratings are quite consistent across the subjects. However it is interesting to analyze the size of the confidence intervals more closely. For most scenes the confidence intervals for the high and low quality levels are smaller than for the middle quality levels. Discussions with the subjects after the test lead to the conclusion that it is very hard to distinguish between intermediate 3D qualities. As expected the influence of the camera distance on the quality of the stereoscopic quality is largely scene-dependent. While for some scenes (sofa, feet, hallway, notebook) the 3D quality decreases considerably with increasing camera distance, it stays nearly constant for other scenes (bike, car). A closer look at the individual curves reveals four different groups: the quality of the first group (bike, car) is between "good" and "excellent" for all the considered camera distances, the second group (sofa) covers the quality range between "fair" and "good", the third group (feet, notebook) covers the whole quality range, and for the fourth group (corridor) the quality is between "bad" and "good". Although a large range of camera distances has been considered, not all the quality levels are equally 
covered. Especially the quality levels at both ends of the scale are only reached for a few sequences (excellent for bike, notebook, car and bad for corridor and notebook). This shows that for non-expert viewers it is quite difficult to distinguish 5 quality levels (excellent, good, fair, poor, bad) and that 3 quality levels (good, fair, bad) may be more appropriate. The corresponding ranges are shown through the dashed horizontal lines.

\subsection{Statistical significance}

In order to understand if the deviations of MOS values for different camera distances and scenes are statistically significant, the analysis of variance (ANOVA) has been applied. In its simplest form the ANOVA is a statistical test to determine if the means of several groups are all equal, which can be seen as a generalization of the Student's two-sample t-test for more than two groups. Given a score matrix $s_{i j}$ where each column (group) represents the scores for a single test condition $j$ for all subjects $i$, the one-way ANOVA tests the null hypothesis that the scores of the different test conditions are drawn from the same population. Therefore, it computes the $F$-statistic as the ratio between the variance among the group means and the variance of the individual samples. The lower the resulting $p$-value of the test, the more statistically significant is the difference among the group means. A typical value for rejecting the null hypothesis is $p<0.05$.

Since we are not only interested if there are differences among the means but also which pairs of means corresponding to the camera distances (scenes) are statistically different, multiple comparison tests are applied. In contrast to an ordinary t-test where the chance of incorrectly finding a significant difference increases with the number of comparisons, multiple comparison tests provide an upper bound on the probability that any comparison can be incorrectly classified as significant. Given the statistics computed by the ANOVA, the TukeyKramer method computes the difference and the confidence interval for each pair of means based on a studentized range distribution. If the resulting confidence intervals for a certain significance level do not contain a difference of 0 , the pair of means is assumed to be significantly different.

Figure 8 summarizes the results of the statistical significance test between the camera distances for the different scenes by showing the p-value of the one-way ANOVA and the pair-wise results of the multiple comparison test as a pseudocolor plot. A p-value smaller than 0.05 means that the $3 \mathrm{D}$ quality of the corresponding scene is significantly influenced by the camera distance. For the pair-wise comparison a white square shows that there is a significant quality difference due to the camera distance while a black square shows that there is none. The statistical significance tests confirm the visual analysis of the MOS/CI plots in figure 7 . The p-values of all the scenes are below 0.05 which shows that the $3 \mathrm{D}$ quality is significantly affected by the camera distance. However, it is interesting that the p-values vary across the difference scenes. The p-value for the car scene is the highest and quite close to the cut-off value which shows that the influence of the camera distance is much less than for the other scenes. The analysis of the pseudo color plots that summarize the pair-wise comparison between camera distances reveals some interesting results. Increasing the camera distance from $10 \mathrm{~cm}$ to $20 \mathrm{~cm}$ does not lead to a significant quality change for all the scenes apart from feet. On the other hand, the quality of the "feet" scene does not change significantly between $20 \mathrm{~cm}$ and $40 \mathrm{~cm}$. In fact, the quality scores can be grouped into 3 distinct levels (good, fair, poor). This behavior may be caused by the small scene depth of $2 \mathrm{~m}$ in combination with the layered depth structure. The other indoor scenes (sofa, hallway, notebook) show a common behavior. There is usually no significant quality difference between adjacent camera distances (e.g. from $20 \mathrm{~cm}$ to $30 \mathrm{~cm}$ ) apart from the notebook scene. The 3D quality decreases gradually for increasing distances. This behavior may be caused by the medium scene depth of $5-20 \mathrm{~m}$ and the gradual depth structure. Finally, a common behavior can be observed for outdoor scenes (bike, car) which do show only marginal differences between the different camera distances. The reason for that may be due to the large scene depth of $110-140 \mathrm{~m}$ and the gradual depth structure.

In order to further analyze the influence of the scene structure on the 3D quality, figure 9 summarizes the statistical significance test between the different scenes. Already the ANOVA with a $p=0$ shows that the quality differs significantly between the different scenes. Looking at the pair-wise comparisons the previous findings are verified. The hallway scene differs from all the other scenes due to the lowest mean score over all the camera distances. This is caused by the fact that even the smallest of the chosen camera distances is slightly above the maximum theoretical one. Furthermore, the scene contains a lot of vertical edges with high contrasts which makes the fusion of a large screen parallax even more difficult. The other indoor scenes (sofa, feet, notebook) 

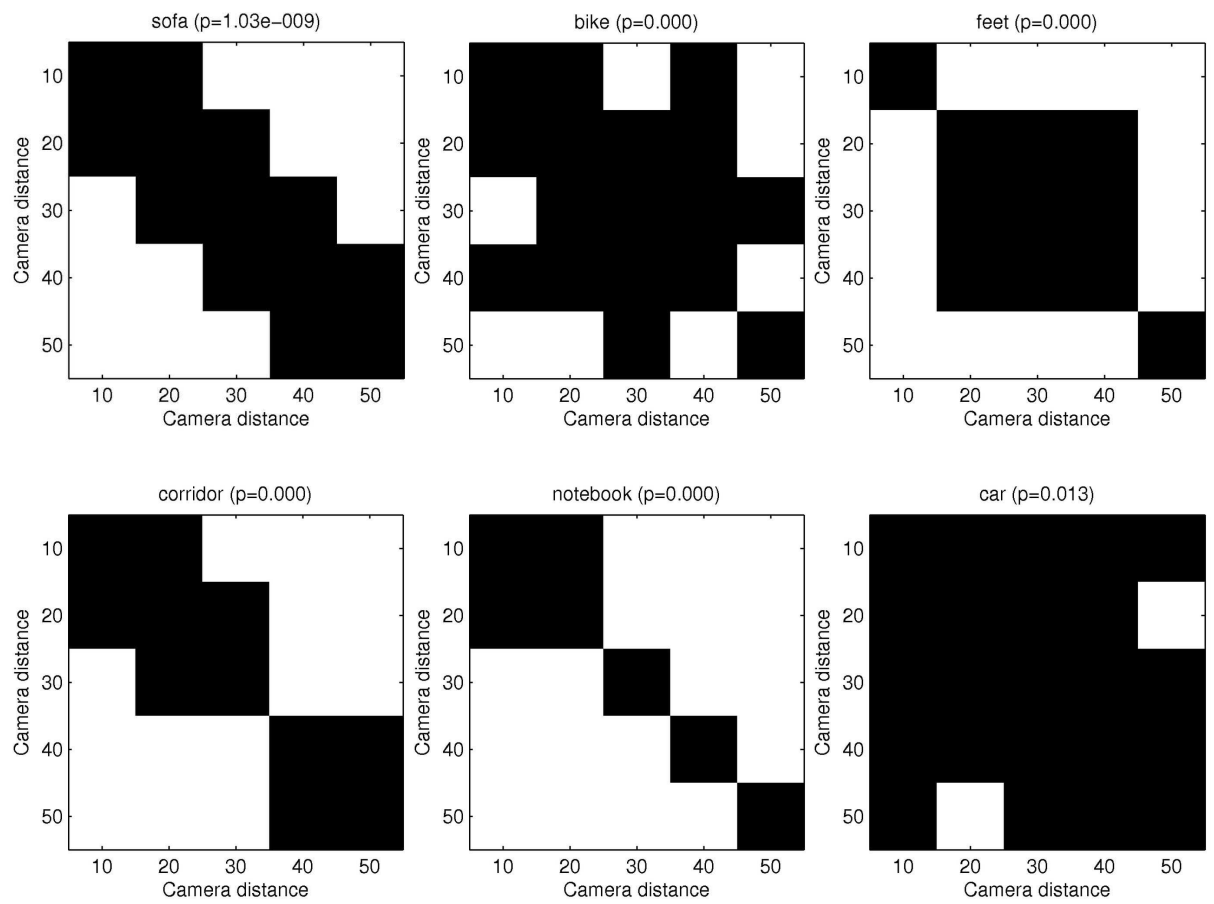

Figure 8: Significant quality deviation between camera distances for the different scenes.

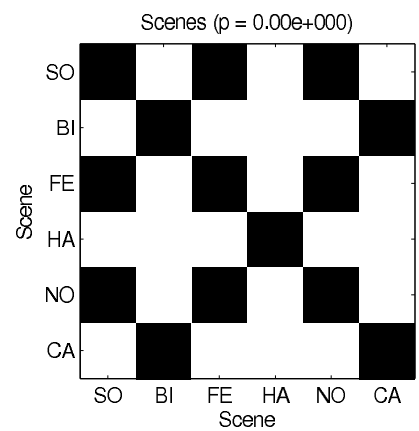

Figure 9: Significant quality deviation between the individual scenes.

do not show any significant quality difference. All of them cover the whole range of quality levels and the global quality distribution is very similar for the different camera distances. The feet scene differs slightly in the medium quality ranges due to the larger amount of motion and the simpler depth structure which makes it difficult to detect small quality differences. Finally, the outdoor scenes (bike, car) differ significantly from the other scenes, since they have a good 3D quality over the whole range of camera distances due to the large scene depth and a lack of vertical edges with high contrasts.

\section{CONCLUSION}

The success of 3DTV will largely depend on the improved quality of experience for the consumer. Since this type of media involves a combination of $2 \mathrm{D}$ and $3 \mathrm{D}$ perception it is important to understand the influence of whole 3D processing chain and the introduced artifacts on the subjective quality. As for the $2 \mathrm{D}$ videos a comprehensive database with associated subjective quality scores is crucial for the optimization of individual processing steps and the development of objective quality metrics of 3DTV. Since already the acquisition of stereoscopic videos may have a large influence on the perceived 3D quality depending a novel database of high definition stereoscopic videos captured with different camera distances has been created. A subjective test has been conducted to quantitatively assess the influence of the camera distance on the perceived quality. It is shown 
that depending on the scene structure and content, subjects are not very sensitive to camera distance changes and that only a few levels of 3D quality can be distinguished. The videos and corresponding subjective quality scores are publicly available

In order to make the database more comprehensible more scenes with different characteristics will be added. Furthermore, new distortions related to the remaining processing steps such as coding, transmission, and restitution will be added. Based on that, it will be possible to compare the influence of different distortions and processing steps on the perceived quality. The resulting subjective scores will be used for the development and evaluation of objective quality metrics for 3DTV.

\section{ACKNOWLEDGMENTS}

The research leading to these results has been performed in the frameworks of European Community's Seventh Framework Program (FP7/2007-2011) under grant agreement no. 216444 (PetaMedia) and Swiss National Centre of Competence in Research (NCCR) on Interactive Multimodal Information Management (IM2).

\section{REFERENCES}

[1] Gotchev, A., Jumisko-Pyykkö, S., Boev, A., and Strohmeier, D., "Mobile 3DTV System: Quality and User Perspective," Proceedings of EUMOB (2008).

[2] MPEG, "Call for contributions on ftv test material," Tech. Rep. N9468, ISO/IEC JTC1/SC29/WG11, Shenzhen, China (Oct 2007).

[3] MPEG, "Call for 3d test material: Depth maps \& supplementary information," Tech. Rep. N10359, ISO/IEC JTC1/SC29/WG11, Lausanne, Switzerland (Feb 2009).

[4] Mendiburu, B., [3D Movie Making], Focal Press (2009).

[5] Hekstra, A., Beerends, J., Ledermann, D., De Caluwe, F., Kohler, S., Koenen, R., Rihs, S., Ehrsam, M., and Schlauss, D., "PVQM - A perceptual video quality measure," Signal processing: Image communication 17(10), 781-798 (2002).

[6] Bercovitz, J., "Image-side perspective and stereoscopy," Proceedings of the SPIE 3295 (1998).

[7] ITU-R, "Methodology for the subjective assessment of the quality of television pictures," Tech. Rep. BT.50011, ITU-R (2002).

[8] ITU-R, "Subjective assessment methods for image quality in high-definition television," Tech. Rep. BT.710-4, ITU-R (1998).

[9] ITU-R, "Subjective assessment of stereoscopic television pictures," Tech. Rep. BT.1438 (2000).

\footnotetext{
${ }^{\ddagger} \mathrm{http}: / / \mathrm{mmspg}$.epfl.ch/3dvqa
} 